\title{
Use of combinatorial pharmacogenomic testing in two cases from community psychiatry
}

This article was published in the following Dove Press journal:

Pharmacogenomics and Personalized Medicine

16 August 2016

Number of times this article has been viewed

\section{Eve S Fields' \\ Raymond A Lorenz ${ }^{2}$ \\ Joel G Winner ${ }^{2}$}

'Northwest Center for Community Mental Health, Reston, VA, USA;

${ }^{2}$ Assurex Health, Mason, OH, USA
Correspondence: Raymond A Lorenz Assurex Health, 6030

Mason-Montgomery Road, Mason,

$\mathrm{OH} 45040$, USA

$\mathrm{Tel}+\mathrm{I} 5137015047$

Fax +I 5134267306

Email rlorenz@assurexhealth.com

\begin{abstract}
This report describes two cases in which pharmacogenomic testing was utilized to guide medication selection for difficult to treat patients. The first patient is a 29 -year old male with bipolar disorder who had severe akathisia due to his long acting injectable antipsychotic. The second patient is a 59-year old female with major depressive disorder who was not responding to her medication. In both cases, a proprietary combinatorial pharmacogenomic test was used to inform medication changes and improve patient outcomes. The first patient was switched to a long acting injectable that was not affected by his genetic profile and his adverse effects abated. The second patient had her medications discontinued due to the results of the genetic testing and more intense psychotherapy initiated. While pharmacogenomic testing may be helpful in cases such as these presented here, it should never serve as a proxy for a comprehensive biopsychosocial approach. The pharmacogenomic information may be selectively added to this comprehensive approach to support medication treatment.
\end{abstract}

Keywords: pharmacogenomics, adverse effects, risperidone, nortriptyline, paliperidone

\section{Introduction}

Patients with mental illness often face a cadre of medication trials before finding a regimen that helps. This trial and error system leaves many patients and providers frustrated and looking for options to assist in medication selection. Combinatorial pharmacogenomic (CPGx) testing has been shown in multiple clinical trials to have clinical validity and utility in psychiatry. ${ }^{1-4}$ Neuropsychiatric medications are metabolized by multiple enzymes and interact with multiple neuropathways in order to attain clinical response. ${ }^{5}$ Pharmacogenomic testing has traditionally made a necessary, but flawed, pharmacologic assumption - that only a single CYP450 enzyme is relevant for a given medication. This assumption was promulgated because the vast number of permutations of genetic effects and metabolic pathways rendered the interpretation of test results unfeasible to implement in clinical practice. CPGx was specifically designed to address this incongruity by integrating results from over 750 published clinical studies, detailed pharmacology, manufacturers' US Food and Drug Administration (FDA)-approved medication labels, and proprietary clinical research to analyze and weight the importance of multiple pharmacokinetic and pharmacodynamic genes. Four possible phenotypes are designated for genes that are part of the CYP450 system: extensive, ultrarapid, intermediate, poor. For a given CYP450 pathway, extensive indicates normal metabolism, ultrarapid indicates faster than normal metabolism, intermediate indicates slower than normal metabolism, and poor indicates little to no 
metabolism. Varying activity of individual enzymes within the CYP450 system may explain the wide range of blood levels for a given medication across patients. We present two cases where CPGx was especially helpful in determining the appropriate medications for these patients. Northwest Center for Community Mental Health has waived the requirement to obtain institutional review board approval for anonymous case reports.

\section{Case presentations and outcome Case I}

This patient was a 29-year old male with a diagnosis of bipolar 1 disorder, most recent episode manic, severe with psychotic features. His recent manic symptoms included agitation, paranoia, irritable mood, and decreased sleep. He presented to the outpatient community mental health center in January 2014 for follow-up. While he was psychiatrically stable, he presented with severe akathisia. There was no known family history of psychiatric illness. Substance use history included current occasional alcohol use and past cannabis use. He was a nonsmoker. Past medical history was unremarkable, except for a history of vitamin D deficiency. The patient's current medication regimen was risperidone long acting injectable $100 \mathrm{mg}$ every 2 weeks. This dose was achieved cautiously over a period of many months because he had been only partially responsive to $50 \mathrm{mg}$ every 2 weeks. The patient did have some mild akathisia at the $50 \mathrm{mg}$ dose, but it was not bothersome at that time. He was also receiving lithium extended-release (ER) 1,200 mg at bedtime, but was chronically non-adherent to the medication and blood level determinations. Previous medication trials included olanzapine, aripiprazole, quetiapine, asenapine, haloperidol, divalproex, and bupropion, all with little efficacy or significant adverse effects.

In order to address his complaints of restlessness, propranolol immediate release was initiated at $20 \mathrm{mg}$ twice daily and titrated to $40 \mathrm{mg}$ twice daily. While receiving propranolol at this dose, his pulse ranged from 46-64 beats per minute, so further increases were deemed unwise despite partial resolution of akathisia. A trial of benztropine was also attempted, but did not help. As part of routine practice at the clinic, pharmacogenomic testing was available to patients based on their clinical presentation, provider preference, and patient willingness. Due to the significant history of failed medications and the severity of his previous manic symptoms, GeneSight Psychotropic pharmacogenomic testing was ordered. GeneSight Psychotropic (Assurex Health, Mason, OH, USA) uses CPGx to classify 38 commonly prescribed antidepressant and antipsychotic medications into three advisory categories based on the level of adverse gene-drug interaction. The three categories are "use as directed" (green), "use with caution" (yellow), and "use with increased caution and more frequent monitoring" (red). At the time of the testing of the patient, six genes were included yielding a report that provides combinatorial, integrated genetic information. These genes include pharmacokinetic genes (CYP2D6, CYP2C19, CYP2C9, CYP1A2) and pharmacodynamic genes (SLC6A4, HTR2A). The following alleles may be detected $C Y P 1 A 2-3860 \mathrm{G}>\mathrm{A}$, $-2467 \mathrm{~T}>\mathrm{delT},-739 \mathrm{~T}>\mathrm{G},-729 \mathrm{C}>\mathrm{T},-163 \mathrm{C}>\mathrm{A}, 125 \mathrm{C}>\mathrm{G}$, $558 \mathrm{C}>\mathrm{A}, 2116 \mathrm{G}>\mathrm{A}, 2473 \mathrm{G}>\mathrm{A}, 2499 \mathrm{~A}>\mathrm{T}, 3497 \mathrm{G}>\mathrm{A}$, $3533 \mathrm{G}>\mathrm{A}, 5090 \mathrm{C}>\mathrm{T}, 5166 \mathrm{G}>\mathrm{A}, 5347 \mathrm{C}>\mathrm{T} ;$ CYP $2 B 6 * 1$, *4, *6, *9; CYP2C19*1, *2, *3, *4, *5, *6, *7, *8, *17; CYP 2 C9*1, *2, *3, *4, *5, *6; CYP $2 D 6 * 1, * 2, * 2 \mathrm{~A}, * 3, * 4$, $* 5, * 6, * 7, * 8, * 9, * 10, * 11, * 12, * 14, * 15, * 17, * 41$, gene duplication; $C Y P 3 A 4 * 1, * 13, * 15 \mathrm{~A},{ }^{*} 2$; HTR $2 A-1438 \mathrm{G}>\mathrm{A}$; SLC6A4 L, S.

Table 1 reports the patient's specific genotype for each of the six genes tested. Interestingly, the patient's GeneSight Psychotropic profile (see Figure 1) showed that many of the medications that had been tried were classified in the yellow or red categories. Based on these results, risperidone long acting injectable (yellow category) was discontinued and paliperidone long acting injectable (green category) was initiated. Lithium was continued. Paliperidone was titrated to $156 \mathrm{mg}$ every 3 weeks and his akathisia reduced significantly over the next few months while his symptoms remained managed.

\section{Case 2}

This patient was a 59-year old woman who presented to the community mental health center in April 2014 with a complaint of severe depression. Her symptoms included depressive ruminations, passive death wish, low energy, poor sleep, and suicidal ideations. The patient had a family history significant for a completed suicide and she had a child with depression and a history of self-injurious behavior. Her past medical history was significant for a total hysterectomy with oophorectomy, obstructive sleep apnea, type 2 diabetes, urinary incontinence, hyperlipidemia, allergic rhinitis, and

Table I Patient I genotype results

\begin{tabular}{lll}
\hline Gene & Genotype & Expected phenotype \\
\hline CYP2D6 & $* 4 / * 5$ & Poor metabolizer \\
CYP2C19 & $* 1 / * 17$ & Ultrarapid metabolizer \\
CYP2C9 & $* 1 / * 1$ & Extensive metabolizer \\
CYPIA2 & $-163 C>A-A / A, 5347 C>$ T - T/T & Ultrarapid metabolizer \\
SLC6A4 & L / S & Moderate response \\
HTR2A & G / G & Reduced activity \\
\hline
\end{tabular}



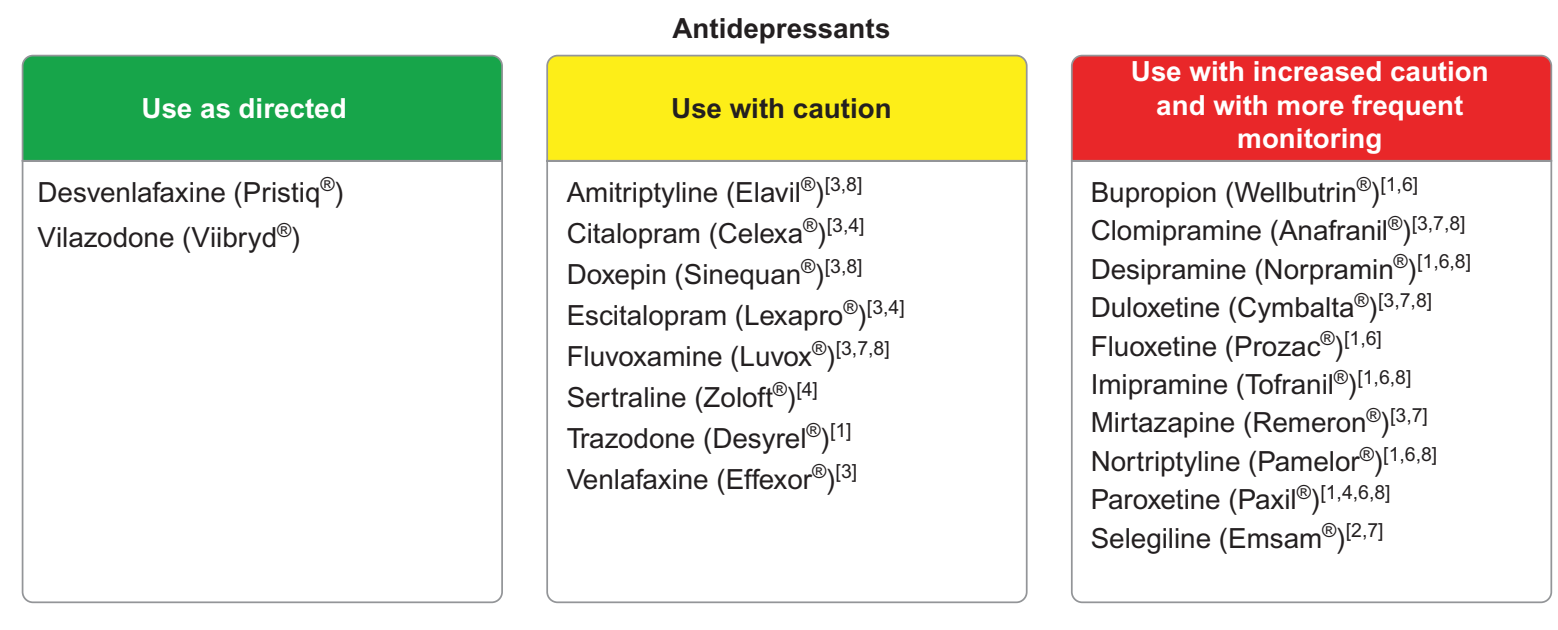

\section{Antipsychotics}

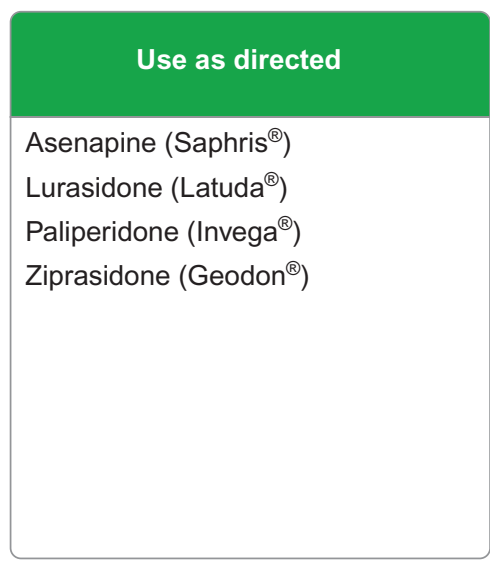

\begin{tabular}{|c|}
\hline Use with caution \\
\hline $\begin{array}{l}\text { Chlorpromazine }\left(\text { Thorazine }^{\circledR}\right)^{[3,7]} \\
\left.\text { Clozapine (Clozaril }{ }^{\circledR}\right)^{[3,7,8]} \\
\left.\text { Fluphenazine (Prolixin }{ }^{\circledR}\right)^{[1]} \\
\left.\text { Olanzapine (Zyprexa }{ }^{[}\right)^{[3,7]} \\
\left.\text { Perphenazine (Trilafon }{ }^{\circledR}\right)^{[3,7,8]} \\
\left.\text { Quetiapine (Seroquel }{ }^{\circledR}\right)^{[1]} \\
\left.\text { Risperidone (Risperdal }{ }^{[囚}\right)^{[1,8]} \\
\left.\text { Thiothixene (Navane }{ }^{\circledR}\right)^{[2,7]}\end{array}$ \\
\hline
\end{tabular}

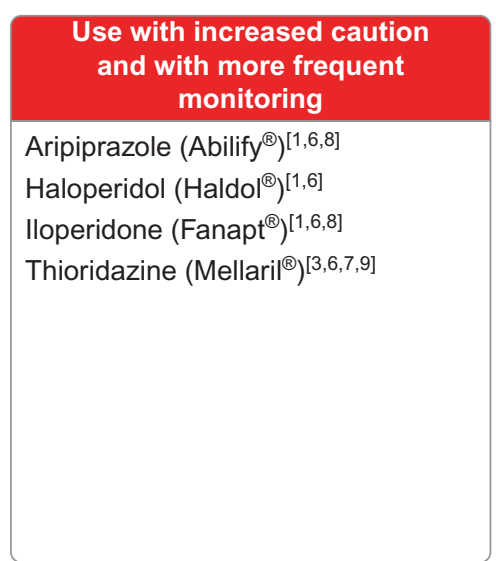

[1]: Serum level may be too high, lower doses may be required.

[2]: Serum level may be too low, higher doses may be required.

[3]: Difficult to predict dose adjustments due to conflicting variations in metabolism.

[4]: Genotype may impact drug mechanism of action and result in reduced efficacy.
[6]: Use of this drug may increase risk of side effects.

[7]: Serum level may be too low in smokers.

[8]: FDA label identifies a potential gene-drug interaction for this medication.

[9]: Per FDA label, this medication is contraindicated for this genotype.

Figure I Patient I GeneSight Psychotropic results.

Note: Reproduced with permission @2016 Assurex Health Inc.

Abbreviation: FDA, US Food and Drug Administration.

a cerebrovascular accident (CVA) over 10 years ago with residual left hemiparesis. Her current medications included trazodone $100 \mathrm{mg}$ po (orally) qhs (every night), gabapentin $300 \mathrm{mg}$ po qhs, glimepiride $2 \mathrm{mg}$ po qday, ranitidine $150 \mathrm{mg}$ po bid (twice daily), and atorvastatin $40 \mathrm{mg}$ po qhs. Her previous psychiatric medication trials included sertraline, duloxetine, desvenlafaxine, and aripiprazole all with minimal efficacy. The patient was diagnosed with major depressive disorder and treatment with citalopram was initiated.

Initially, the patient responded to citalopram, but after 2 months, efficacy decreased and lithium was added as an augmenting agent. The addition was moderately effective, but the patient continued to have passive death wish and depressive ruminations. Citalopram was discontinued and nortriptyline was initiated at $10 \mathrm{mg}$ at bedtime. This lower dose was chosen due to the patient's history of CVA. After 1 month, lithium and nortriptyline blood concentrations were ordered and were $0.6 \mathrm{mEq} / \mathrm{L}$ and $0 \mathrm{ng} / \mathrm{mL}$ respectively. Patient endorsed current adherence with her medications evidenced by her lithium concentration, but did have a history of non-adherence. Pharmacogenomic testing was ordered to determine if the patient's genetic profile could be contributing to the lack of efficacy of her medications. Additionally, the genetic results had the potential to explain the discrepancy between the patient's nortriptyline serum levels and her claim that she was adherent with the medication.

Table 2 reports the patient's specific genetic genotype for each of the six genes tested. Many of the patient's previous medications were in the yellow or red category, including the nortriptyline she was currently receiving (Figure 2). 
Nortriptyline was initially increased to $25 \mathrm{mg}$ at bedtime, but was stopped after 2 months when there was no evidence of efficacy. Further titration of nortriptyline was not performed due to her fragile medical state post-CVA and concerns about potential risk for falls. Despite probable adherence, the increased dose was not supplying adequate blood levels (nortriptyline level was still undetectable). The lithium was continued, the patient's psychotherapy was intensified, and

Table 2 Patient 2 genotype results

\begin{tabular}{lll}
\hline Gene & Genotype & Expected phenotype \\
\hline CYP2D6 & $* I / * I$ with duplication & Ultrarapid metabolizer \\
CYP2CI9 & $* I / * I$ & Extensive metabolizer \\
CYP2C9 & $* I / * I$ & Extensive metabolizer \\
CYPIA2 & $-3860 G>A-A / A,-2467 T>D E L T-$ & Ultrarapid metabolizer \\
& DELT/DELT, - I63C $>$ A - A/A & \\
SLC6A4 & S / S & Reduced response \\
HTR2A & G / A & Intermediate activity \\
\hline
\end{tabular}

additional psychosocial interventions such as home visits were used. Due to pharmacogenomic testing, the patient's medication regimen was able to be simplified and adverse outcomes such as falls were avoided.

\section{Discussion}

Patient 1 had improvement of manic symptoms on high dose risperidone, however akathisia complicated treatment and adherence. Risperidone is converted to 9-hydroxyrisperidone (paliperidone) primarily by CYP2D6 and to a lesser extent by CYP3A4. Risperidone and paliperidone plasma concentrations were not obtained in this patient due to testing being cost prohibitive. The patient had no functioning CYP2D6 alleles - making him a quintessential poor metabolizer, which in combination with high doses of depot risperidone, likely combined to produce akathisia in this patient. Compounding this problem, the patient's akathisia was treated with pro-
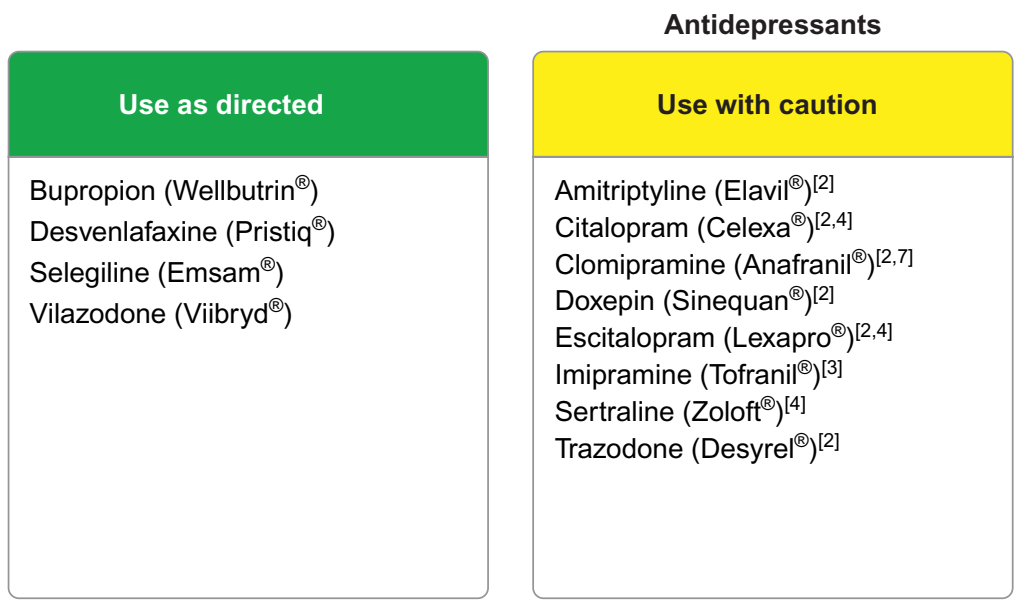

Antipsychotics
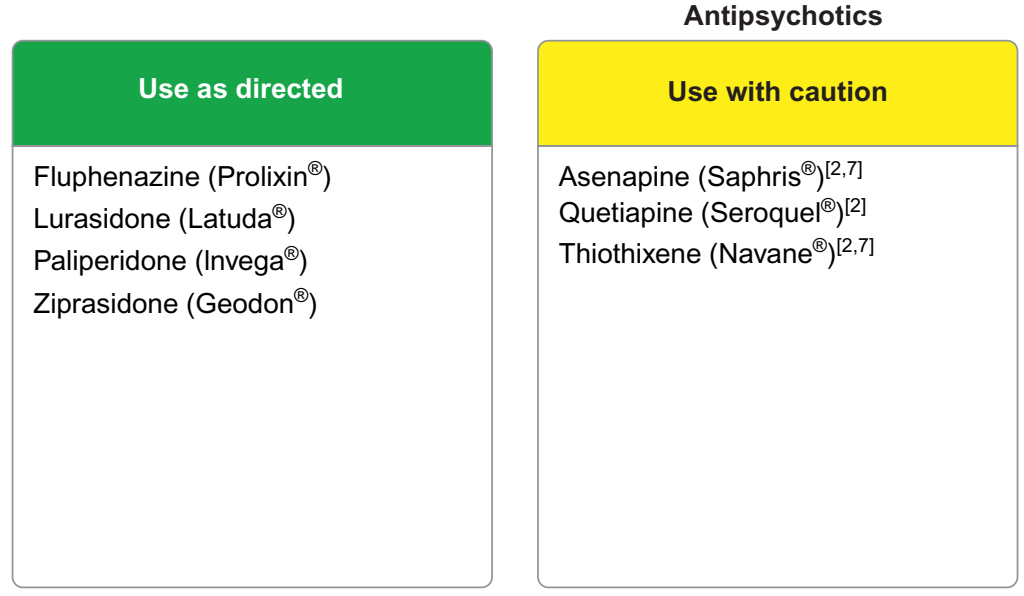

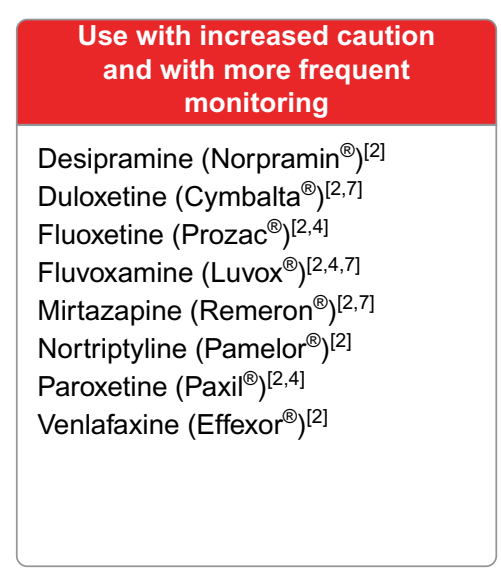

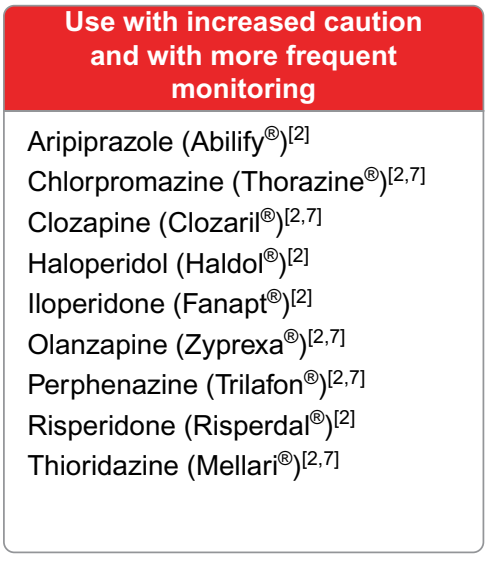

[2]: Serum level may be too low, higher doses may be required.

[3]: Difficult to predict dose adjustments due to conflicting variations in metabolism.
[4]: Genotype may impact drug mechanism of action and result in reduced efficacy.

[7]: Serum level may be too low in smokers. 
pranolol, which is also metabolized by CYP2D6, possibly contributing to the patient's low heart rate. When the patient was switched to 9-hydroxyrisperidone (paliperidone), he achieved efficacy without major side effects. Paliperidone, unlike risperidone, is not metabolized by CYP2D6. As such, paliperidone concentrations would not be affected by this patient's genetic polymorphism in CYP2D6. In fact, the FDA package insert for paliperidone states ${ }^{6}$, "population pharmacokinetics analyses indicated no discernible difference on the apparent clearance of paliperidone after administration of oral paliperidone between extensive metabolizers and poor metabolizers of CYP2D6 substrates." Further, paliperidone may have a lower risk of akathisia compared to risperidone.

Some clinicians erroneously believe that depot forms of medication are not metabolized by the $\mathrm{P} 450$ system. While depot medications largely skip first pass metabolism, ${ }^{8}$ they are still ultimately metabolized by CYP enzymes, which inform steady-state concentrations. ${ }^{9}$ Additionally, some studies have shown that patients with the G/G genotype at the HTR2A gene may have an increased response to risperidone. ${ }^{10-15}$ So while this patient may have had a better response to risperidone based on this genotype, dose-related adverse effects complicated his treatment highlighting that genes cannot be used in isolation to determine medication treatment and should be considered in combination. No literature exists looking at response to paliperidone and the HTR2A gene.

Patient 2 was adherent to her medication regimen, but was shown to be an ultrarapid metabolizer at CYP2D6 leading to lower than expected blood levels. Furthermore, the narrow therapeutic index for tricyclic antidepressants makes patients who receive them ideal candidates for pharmacogenomic testing. Even at low doses, a patient who is a poor metabolizer for a given substrate medication could have higher than anticipated serum levels which may lead to toxicity. In this case, the patient had increased enzyme activity for the primary metabolic pathway for nortriptyline which caused an undetectable serum level. Without a detectable serum level, there is no potential for a medication-mediated improvement in symptoms. With pharmacogenomic testing, it was determined that the low level could be attributed to increased enzyme activity and not necessarily poor adherence. Serum level monitoring of tricyclic antidepressants provides clinical information for a snapshot in time. However, combining this strategy with pharmacogenomic testing can provide a more complete picture of the disposition of the medication, enable more accurate dosing, and most importantly give insight into possible future treatment strategies.
Using CPGx to guide medication selection for the cases presented here was valuable and informative. Patients who have failed medications due to ineffectiveness or adverse events and have been deemed treatment-resistant may benefit from CPGx. GeneSight Psychotropic has shown clinical validity and clinical utility in clinical trials, but also cost effectiveness. ${ }^{16}$ When used appropriately and in combination with clinical judgment, pharmacogenomic testing can be a powerful tool in selected circumstances to help patients achieve wellness.

\section{Disclosure}

No financial or material support was received for this work. Both Dr Lorenz and Dr Winner are employees of Assurex Health. The authors have no other conflict of interest to disclose.

\section{References}

1. Hall-Flavin DK, Winner JG, Allen JD, et al. Using a pharmacogenomic algorithm to guide the treatment of depression. Transl Psychiatry. 2012;2:e172.

2. Hall-Flavin DK, Winner JG, Allen JD, et al. Utility of integrated pharmacogenomic testing to support the treatment of major depressive disorder in a psychiatric outpatient setting. Pharmacogenet Genomics. 2013; 23(10):535-548.

3. Winner JG, Carhart JM, Altar CA, Allen JD, Dechairo BM. A prospective, randomized, double-blind study assessing the clinical impact of integrated pharmacogenomic testing for major depressive disorder. Discov Med. 2013;16(89):219-227.

4. Altar CA, Carhart JM, Allen JD, Hall-Flavin DK, Dechairo BM, Winner JG. Clinical validity: Combinatorial pharmacogenomics predicts antidepressant responses and healthcare utilizations better than single gene phenotypes. Pharmacogenomics J. 2015;15(5):443-451.

5. Mrazek DA. Psychiatric Pharmacogenomics. Oxford University Press; 2010.

6. INVEGA $®$ (paliperidone) extended-release tablets [package insert]. Titusville, NJ: Janssen Pharmaceuticals; 2015.

7. Suzuki H, Gen K, Inoue Y, et al. The influence of switching from risperidone to paliperidone on the extrapyramidal symptoms and cognitive function in elderly patients with schizophrenia: a preliminary open-label trial. Int J Psychiatry Clin Pract. 2014;18(1):58-62.

8. Altamura AC, Sassella F, Santini A, Montresor C, Fumagalli S, Mundo E. Intramuscular preparations of antipsychotics: uses and relevance in clinical practice. Drugs. 2003;63(5):493-512.

9. Choong E, Polari A, Kamdem RH, et al. Pharmacogenetic study on risperidone long-acting injection: influence of cytochrome P450 2D6 and pregnane $\mathrm{X}$ receptor on risperidone exposure and drug-induced side-effects. J Clin Psychopharmacol. 2013;33(3):289-298.

10. Lane HY, Chang YC, Chiu CC, Chen ML, Hsieh MH, Chang WH. Association of risperidone treatment response with a polymorphism in the 5-HT(2A) receptor gene. Am J Psychiatry. 2002;159(9): 1593-1595.

11. Yamanouchi Y, Iwata N, Suzuki T, Kitajima T, Ikeda M, Ozaki N. Effect of DRD2, 5-HT2A, and COMT genes on antipsychotic response to risperidone. Pharmacogenomics J. 2003;3(6):356-361.

12. Alenius M, Wadelius M, Dahl M-L, Hartvig P, Lindström L, Hammarlund-Udenaes M. Gene polymorphism influencing treatment response in psychotic patients in a naturalistic setting.JPsychiatr Res. 2008; 42(11):884-893. 
13. Ikeda M, Yamanouchi Y, Kinoshita Y, et al. Variants of dopamine and serotonin candidate genes as predictors of response to risperidone treatment in first-episode schizophrenia. Pharmacogenomics. 2008; 9(10):1437-1443.

14. Kim B, Choi EY, Kim CY, Song K, Joo YH. Could HTR2A T102C and DRD3 Ser9Gly predict clinical improvement in patients with acutely exacerbated schizophrenia? Results from treatment responses to risperidone in a naturalistic setting. Hum Psychopharmacol. 2008;23(1): 61-67.
15. Angelucci F, Bernardini S, Gravina P, et al. Delusion symptoms and response to antipsychotic treatment are associated with the 5-HT2A receptor polymorphism $(102 \mathrm{~T} / \mathrm{C})$ in Alzheimer's disease: a 3-year follow-up longitudinal study. J Alzheimers Dis. 2009;17(1):203-211.

16. Winner J, Lorenz RA, Allen JD, Carhart JM, Dechairo BM. Health Care Utilization and Medication Cost Savings in Patients Receiving Combinatorial Psychiatric Pharmacogenomic Testing. Poster presented at: American Psychiatric Association Annual Meeting; May 18, 2015; Toronto, ON.

\section{Publish your work in this journal}

Pharmacogenomics and Personalized Medicine is an international, peerreviewed, open access journal characterizing the influence of genotype on pharmacology leading to the development of personalized treatment programs and individualized drug selection for improved safety, efficacy and sustainability. This journal is indexed on the American Chemical
Society's Chemical Abstracts Service (CAS). The manuscript management system is completely online and includes a very quick and fair peer-review system, which is all easy to use. Visit http://www.dovepress. com/testimonials.php to read real quotes from published authors. 